\title{
The Rho GTPase RhoE is a p53-regulated candidate tumor suppressor in cancer cells
}

\author{
YAJIE ZHU $^{1 *}$, JITAO ZHOU $^{1 *}$, HONGWEI XIA $^{1^{*}}$, XIANGZHENG CHEN $^{1}$, MENG QIU $^{1}$, JUAN HUANG $^{1}$, \\ SURUI LIU ${ }^{1}$, QIULIN TANG ${ }^{1}$, NAN LANG ${ }^{1}$, ZHEN LIU $^{1}$, MING LIU $^{1}$, YI ZHENG $^{2}$ and FENG BI ${ }^{1}$ \\ ${ }^{1}$ Department of Medical Oncology and Laboratory of Signal Transduction and Molecular Targeting Therapy, \\ West China Cancer Center, West China Hospital, Sichuan University, Chengdu, Sichuan 610041, P.R. China; \\ ${ }^{2}$ Division of Experimental Hematology and Cancer Biology, Children's Hospital Research Foundation, \\ University of Cincinnati, Cincinnati, OH 45229, USA
}

Received November 1, 2013; Accepted December 12, 2013

DOI: $10.3892 /$ ijo.2014.2245

\begin{abstract}
Previous studies have shown that RhoE, an atypical member of the Rho GTPase family, may play an opposite role to RhoA in regulating cell proliferation and invasion. To explore the relationship between RhoE and the malignant phenotypes of human cancer, we have determined the expression patterns of RhoE in varying grade of human cancer tissues and tested the effects of RhoE expression in several RhoE underexpressing cancer cell lines. Systemic immunocytochemistry analyses of gastric, colorectal, lung and breast carcinomas, respectively, showed that RhoE protein expression was significantly decreased in most cancer cases compared with that of adjacent normal tissues. Enhanced RhoE expression could markedly inhibit proliferation, migration and invasion and induce apoptosis of the cancer cells which have relatively low levels of endogenous RhoE expression. Wild-type p53 (wt-p53) could strongly increase RhoE expression in p53-transfected cells. Furthermore, the luciferase assays indicated that wt-p53 significantly enhanced the activities of RhoE promoter compared with mutant p53 (mt-p53) in PC3 cells (p53 null). Collectively, data are presented showing that RhoE may participate in human
\end{abstract}

Correspondence to: Professor Feng Bi, Department of Medical Oncology, West China Hospital, Sichuan University, Chengdu, Sichuan 610041, P.R. China

E-mail: bifeng@medmail.com.cn

${ }^{*}$ Contributed equally

Abbreviations: Rho, Ras homology; ROCK, Rho-associated coiledcoil forming protein serine-threoine protein kinases; SDS, sodium dodecyl sulfate; PAGE, polyacylamide gel electrophoresis cdc division cycle

Key words: Rho GTPases, RhoE, p53, cancer, tumor suppressor, invasion, cell cycle cancer progression and act as a candidate target of p53, and these findings also strongly suggest that RhoE may be a new candidate tumor suppressor and could serve as a potential target in the gene therapy of cancer.

\section{Introduction}

Rho family GTPases, a part of the Ras superfamily which are involved in regulating multiple aspects of cell functions such as cell proliferation, survival, adhesion and migration, are intimately relevant in tumor initiation and tumor progression (1-5). Most members of the Rho family can cycle between an active, GTP-bound conformation and an inactive, GDP-bound conformation, and this GTP-binding and GTP-hydrolysis process is tightly regulated by various classes of regulators including the guanine nucleotide exchange factors and GTPase-activating proteins. The best characterized members of the Rho family include Rho (A, B and C), $\operatorname{Rac}(1,2$ and 3) and Cdc42. RhoE is a unique member of the Rnd subfamily, a distinct branch of Rho family proteins that bind, but do not hydrolyze GTP, thus remains constitutively active upon expression without the regulation by guanine nucleotide exchange factors and GTPase-activating proteins (6-9). In addition, the carboxy-terminal sequence of RhoE is farnesylated while most Rho family proteins are modified by geranylation. These observations suggest that the function and regulation of RhoE may be different from other Rho family members (6).

There are some studies on the role of RhoE in human malignancies. Several reports have examined RhoE expression patterns in tumor tissues, but the results were contradictory (7,10-12). RhoE expression level does not change in response to serum stimulation, however, it can be enhanced by DNA-damaging agents such as cisplatin or UBV (9). Tumor suppressor p53 can also induce cell cycle arrest at G1 and allow cells to repair DNA damage induced by ionizing radiation, UV light or chemotherapeutic drugs before proceeding to DNA replication (13-15). Ongusaha et al have shown that RhoE is a transcriptional target of p53 and can inhibit ROCK1mediated apoptosis in response to genotoxic stress (16). 
In the present study we systematically investigated the expression levels of RhoE in human gastric, colorectal, lung and breast cancer tissues by using tissue microarrays and explored the effect of RhoE expression on the growth and invasion phenotypes of the cancer cell lines. We further attribute the relatively reduced RhoE expression to the reduction of $\mathrm{p} 53$ transcription activity in the cancer cells and present evidence that RhoE expression could be affected by p53 mutation. These findings may partly explain the mechenism of RhoE downregulation in some cancers.

\section{Materials and methods}

Cell culture. Human embryonic kidney cells 293, gastric cancer cell lines MKN45 and AGS, colorectal cancer cell lines SW480 and LOVO, liver cancer cell lines HepG2 and SMMC7721, lung cancer cell lines A549 and SPCA1, and the prostate carcinoma cell line PC3, were preserved at our institute. All cell lines were maintained in RPMI-1640 medium (Invitrogen Corporation, Gaithersburg, MD, USA) supplemented with $10 \%$ fetal bovine serum (FBS) (Gibco, Carlsbad, CA, USA), $100 \mathrm{U} / \mathrm{ml}$ penicillin $\mathrm{G}$ sodium, and $100 \mu \mathrm{g} / \mathrm{ml}$ streptomycin sulfate (Sigma, St Louis, MO, USA). Cells were grown at $37^{\circ} \mathrm{C}$ in a humidified atmosphere containing $5 \% \mathrm{CO}_{2}$.

Plasmids and transfection. Expression vectors pCDNA3.0RhoE, PEGFP, MIEG3-wt-p53, p21-promoter-luc and PRL-TK were kindly provided by Professor Yi Zheng (Cincinnati Children's Hospital, Cincinnati, OH, USA). MIEG3-wt-p53 and PEGFP were digested with BamH1 and EcoR1 in order to construct the expression vector PEGFP-wt-p53. PEGFPmt-p53 plasmids that mutated at p53 hot points $(175,248,273$, $282,175 / 248,175 / 273,175 / 282,248 / 273,248 / 282,273 / 282$ ) were constructed on the basis of PEGFP-wt-p53. PGL3-RhoEpromoter-3000-luc was stored in our laboratory. Cells were transfected by Lipofectamine 2000 (Invitrogen), and stable transfection cell lines were selected with G418 (Gibco).

Immunohistochemistry. Tissue microarrays were obtained from Cybrdi Co. (Xi'an, China), containing 74 cases of gastric carcinoma, 32 cases of colorectal carcinoma, 62 cases of lung carcinoma and 34 cases of breast carcinoma. All were matched with the adjacent cancerous tissues and their clinicopathological grades were evaluated using WHO standards. The tissue microarrays were evaluated by three pathologists who did not know the information of the slides. IHC studies were performed using a standard avidin-biotin complex immunoperoxidase method. Briefly, tissue sections were deparaffinized in xylene and rehydrated with a series of decreasing alcohol concentrations and the endogenous peroxidase activity was inhibited with a $3 \%$ solution of $\mathrm{H}_{2} \mathrm{O}_{2}$ for $10 \mathrm{~min}$. For antigen retrieval, tissue slides were treated in citrate buffer ( $\mathrm{pH}$ 6.0) with microwave for $10 \mathrm{~min}$. The detection was then performed following the instruction of mouse streptavidin-peroxidase immunostaining kit (SP-9000 Histostain-Plus Kit; Zhong Shan Goldbridge Biotechnology Co., Ltd, Beijing, China) containing normal goat serum, biotinylated secondary antibody and streptavidinperoxidase complex. The slides were independently incubated with anti-RhoE monoclonal antibody (05-723, clone 4, Upstate Biotechnology, Lake Placid, NY, USA) which were diluted
1:50 in PBS overnight at $4^{\circ} \mathrm{C}$. Pre-immune serum, instead of a primary antibody, was used as a negative control. Then the slides were stained with DAB (Pierce Biotechnology, Rockford, IL, USA), dehydrated, cleared and mounted with neutral balsam.

The ratio of positive cells per specimen was evaluated quantitatively and scored: 0 for staining $\leq 1 \%, 1$ for staining of 2 to $25 \%, 2$ for staining of 26 to $50 \%, 3$ for staining of 51 to $75 \%$, and 4 for staining $>75 \%$ of the cells examined. Staining intensity was graded as follows: 0 , no signal; 1 , weak; 2 , moderate; and 3, strong staining. Both scoring systems were utilized to evaluate the level of protein expression. A total score of 0 to 12 was finally calculated and graded as negative (-, $0-1)$; weak $(+, 2-4)$; moderate $(++, 5-8)$; and strong $(+++, 9-12)$. This scoring system has been described previously.

Monolayer growth rate and cell cycle assay. Cells (500-1,000) were plated in 96-wells cell culture plate. Cell counting kit-8 (Dojindo, Kumamoto, Japan) was used to determine the monolayer culture growth rate. Cultures were assayed at 0 , $1,2,3,4,5$ or 6 days. Absorbance values were determined on the microplate reader (Bio-Rad Laboratories, Hercules, CA, USA) at 490 and $630 \mathrm{~nm}$. Cell cycle profiles were measured by flow cytometry using propidium iodide (PI). Transfectants which were stable transfected with RhoE and pcDNA3.0, respectively, were starved for $24 \mathrm{~h}$ in $0.5 \%$ serum-containing medium and then were stimulated with $10 \%$ FBS before fixing with $70 \%$ ethanol at $4^{\circ} \mathrm{C}$ overnight, washed with PBS, and stained with PI $(50 \mu \mathrm{g} / \mathrm{ml})$ in PBS supplemented with RNase $(10 \mathrm{mg} / \mathrm{ml})$ for $30 \mathrm{~min}$. Flow cytometry was performed on a FACScan (Becton-Dickinson, Heidelberg, Germany) equipped with cellquest software, and cellular DNA content was determined for $1 \times 10^{4}$ cells.

In vitro apoptosis assay. Hoechst/PI staining and TUNEL experiments were performed to study cell apoptosis. First, cells were trypsinized and washed with PBS twice. For the Hoechst/PI staining, prepared cells were fixed in $100 \mu \mathrm{l}$ PBS containing $0.1 \mu \mathrm{g}$ Hoechst 33342 for $10 \mathrm{~min}$ at $37^{\circ} \mathrm{C}$. After centrifuging, cells were stained with PI. Next, the apoptotic cells were viewed and counted by fluorescent microscopy at once. For the TUNEL experiment, $1 \times 10^{5}$ prepared cells were added into 6 -wells cell culture plates which were coated with thin slides. Cells were harvested for $48 \mathrm{~h}$ and then tested by chromogenic method TUNEL apoptosis detection kit (Beyotime, Jiangsu, China). Cells were fixed in $4 \%$ paraformaldehyde for $1 \mathrm{~h}$, and then incubated in Biotin-UTP solution for $1 \mathrm{~h}$ at room temperature. After that, samples were incubated in streptavidin-HRP buffer and colored by DAB and hematoxylin. TUNEL-positive nuclei stained dark brown and TUNEL-negative nuclei stained blue. The sections were examined using light microscopy.

Invasion assay. Millicell hanging cell culture inserts (Millipore, Billerica, MA, USA) precoated with Matrigel (Becton-Dickinson) were put in a 24 -well plate to perform

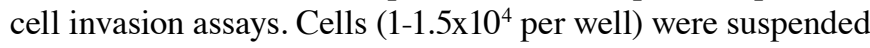
in $0.25 \mathrm{ml}$ of culture medium with $1 \% \mathrm{FBS}$ and added to the upper chamber. Medium $(1.25 \mathrm{ml})$ supplemented with $10 \%$ FBS was plated in the bottom of the well. The invasion 
assay was carried out for $48 \mathrm{~h}$ in a humidified incubator. The cells that traversed the membrane pore and spread to the lower surface of the filters were stained with eosin for visualization and then counted in 10 random high power fields (x200).

Western blot analysis. Cells were lysed in lysis buffer containing $50 \mathrm{mM}$ Tris- $\mathrm{HCl}$ (pH 7.4), $150 \mathrm{mM} \mathrm{NaCl}, 0.2 \mathrm{~g} / \mathrm{l}$ sodium azide, $1 \mathrm{~g} / 1 \mathrm{SDS}, 1 \%$ Triton $\mathrm{X}-100$, together with a protease inhibitor mix (Calbiochem, La Jolla, CA, USA). Cellular protein was quantified by a BCA protein assay kit (Pierce). Equal amounts of proteins (30-40 $\mu \mathrm{g})$ were fractionated by $8-15 \%$ sodium dodecyl sulfate-polyacrylamide gels (SDS-PAGE) and then transferred to PVDF (Millipore). The sheets were pre-incubated in a mixture of PBS and 5\% non-fat milk powder for $2 \mathrm{~h}$ at room temperature. For immunoblotting studies, membranes were incubated overnight at $4^{\circ} \mathrm{C}$ with $5 \%$ non-fat milk powder containing the primary antibodies as following: antibodies against RhoE (05-723, clone 4, Upstate Biotechnology). After a TBS- $0.05 \%$ Tween-20 wash, the sheets were incubated with an IRDye $800 \mathrm{CW}$ secondary antibody (LI-COR Biosciences, Lincoln, NE, USA) at room temperature for $1 \mathrm{~h}$ in a cassette. After incubation, the sheets were washed in TBS-0.05\% Tween-20 also in a cassette and then detected by Odyssey infrared fluorescence scanner (LI-COR).

Dual-luciferase reporter assay. For dual-luciferase reporter assays, $1 \times 10^{5}$ cells per well in 24 -well plates were seeded the day before transfection. Cells were transfected with $1.0 \mu \mathrm{g}$ of p21-promoter-luc together with $0.2 \mu \mathrm{g}$ PRL-TK or RhoE-promoter-luc $0.4 \mu \mathrm{g}$, wt-p53/mt-p53 $1.0 \mu \mathrm{g}$ together with $0.2 \mu \mathrm{g}$ PRL-TK. The cells were harvested for $48 \mathrm{~h}$ and then detected by dual-luciferase reporter assay system kit (Promega, Madison, WI, USA). The luciferase activity was measured with the Synergy2 instrument (BioTek, Winnoski, VT, USA) equipped with Gen5 software. The firely luciferase expression was normalized to Renilla luciferase and reported as relative luciferase activity. Results are presented as means \pm standard deviations (SD) of data from three dependent experiments.

RNA extraction and p53 mutation detection. The total RNA of 293, MKN45, AGS, SW480, LOVO, SPCA1, A549, HepG2 and SMMC-7721 was extracted using TRIzol (Invitrogen) according to the manufacturer's protocol. The quantity and purity of the RNA was determined by electrophoresis and the ratio of the optical density at $260 \mathrm{~nm}$ to that at $280 \mathrm{~nm}$. p53 was amplified by RT-PCR using the SuperScript ${ }^{\mathrm{TM}}$ III One-step RT-PCR system with Platinum ${ }^{\circledR}$ Taq high fidelity kit (Invitrogen). Primers used for p53 were: forward, 5'-AGTCTAGAGCCACCGTCCA-3'; and reverse, 5'-TCT GACGCACACCTATTGCAAGC-3' (17). A total of $2 \mu \mathrm{l}$ RNA was brought to a volume of $50 \mu \mathrm{l}$ containing $25 \mu \mathrm{l} 2 \mathrm{X}$ reaction mix, $1 \mu \mathrm{l}$ SuperScript/Platinum Taq high fidelity enzyme mix, $1 \mu 1$ of both sense and antisense primer. Amplification was performed as per the following conditions: one cycle of $55^{\circ} \mathrm{C}$ for $30 \mathrm{~min}$ was performed to synthesize the cDNA. After denaturation at $94^{\circ} \mathrm{C}$ for 2 min 40 cycles of PCR were performed to amplify p53 (denaturation at $94^{\circ} \mathrm{C}$ for $15 \mathrm{sec}$, annealing at $58^{\circ} \mathrm{C}$ for $30 \mathrm{sec}$, extension at $68^{\circ} \mathrm{C}$ for $90 \mathrm{sec}$ and a final extension of $68^{\circ} \mathrm{C}$ for $5 \mathrm{~min}$ ). The PCR products were analyzed by agarose gel electrophoresis.
The p53 amplicons were purified and linked with A tail by DNA A-tailing kit (Takara Bio, Dalian, China), then subcloned into pMD18-T vector. The DNAs were sequenced by using vector primers (Invitrogen).

Statistical analysis. SPSS11.0 software package (SPSS, Chicago, IL, USA) was used to analyze data. Pared-samples t-test was used to compare the stained value of the immunohistochemistry samples. The correlation between tumor grades and the stained value and the positive ratio were tested by $\chi^{2}$ test. The correlation of molecules was tested by multiple linear regression analysis. Assays for characterizing phenotype of cells were analyzed by Student's t-test. A value of $\mathrm{p}<0.05$ was considered to indicate statistical significance.

\section{Results}

Reduced expression of RhoE in human gastric, colorectal, lung and breast cancer tissues. Our laboratoty, previously, has reported that RhoA and RhoC expressions are significantly upregulated in gastric cancer tissues, and RhoE expression is likely regulated by epigenetic modification. We performed immunohistochemistry staining of cancer tissue microarrays that contain 74 gastric, 32 colorectal, 62 lung and 34 breast cancer carcinoma samples matched with adjacent normal tissues, respectively, in an effort to examine the protein expression of RhoE in human cancer tissues. The staining examination of the tumor tissues in comparison with the adjacent normal tissues showed that RhoE was abundantly expressed in the cytoplasm of the normal tissues but is at a low or non-detectable level in the cancer tissues in general (Fig. 1A). We have attempted to quantify the relative RhoE expression levels based on parallel immunohistochemistry staining of the array chips by assigning a score ranging from '-', '+', ' ++ ' to ' +++ ' that correspond to $1-4$ in a blank-folded manner. This exercise yielded average staining scores of $1.99 \pm 0.28$ (positive ratio was $41.9 \%$ ), $2.45 \pm 0.35$ (positive ratio was $68.7 \%$ ), $2.19 \pm 0.19$ (positive ratio was $71.0 \%$ ) and $3.65 \pm 0.62$ (positive ratio was $58.8 \%$ ) for gastric, colorectal, lung and breast cancers, respectively, while the average staining scores of the adjacent tissues was 8.64 \pm 0.39 (positive ratio was 94.6\%), 6.55 \pm 0.63 (positive ratio was 90.6\%), $4.11 \pm 0.24$ (positive ratio was 93.5\%), 10.53 \pm 0.44 (positive ratio was $100.0 \%$ ). The differences of the staining scores and positive ratios between the respective tumor tissues and the adjacent tissues were highly significant (p<0.005) (Fig. 1B). Therefore, RhoE expression level appears to be inversely correlated with tumorigenesis in human gastric, colorectal, lung and breast cancers.

Since a possible divergence of the relative scores is related to different cancer progressive grades, we further examined RhoE expression levels in the tumor tissues based on the available cancer grades from the patient samples. Among the gastric, colorectal and lung cancer patient samples with known cancer grades, RhoE expression correlated well with the cancer grades $(\mathrm{p}<0.005)$. These analyses suggest that $\mathrm{RhoE}$ expression is also associated with cancer progression.

Enhanced RhoE expression inhibits cancer cell proliferation and invasion. The expression level of $\mathrm{RhoE}$ was investigated 
A
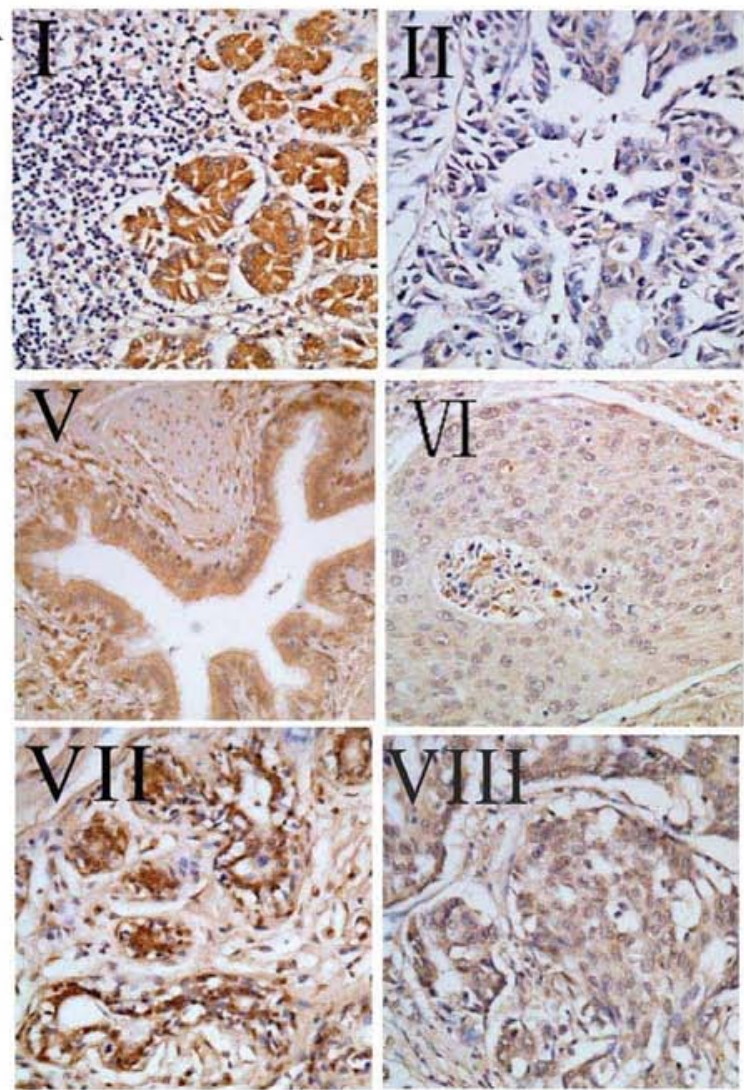
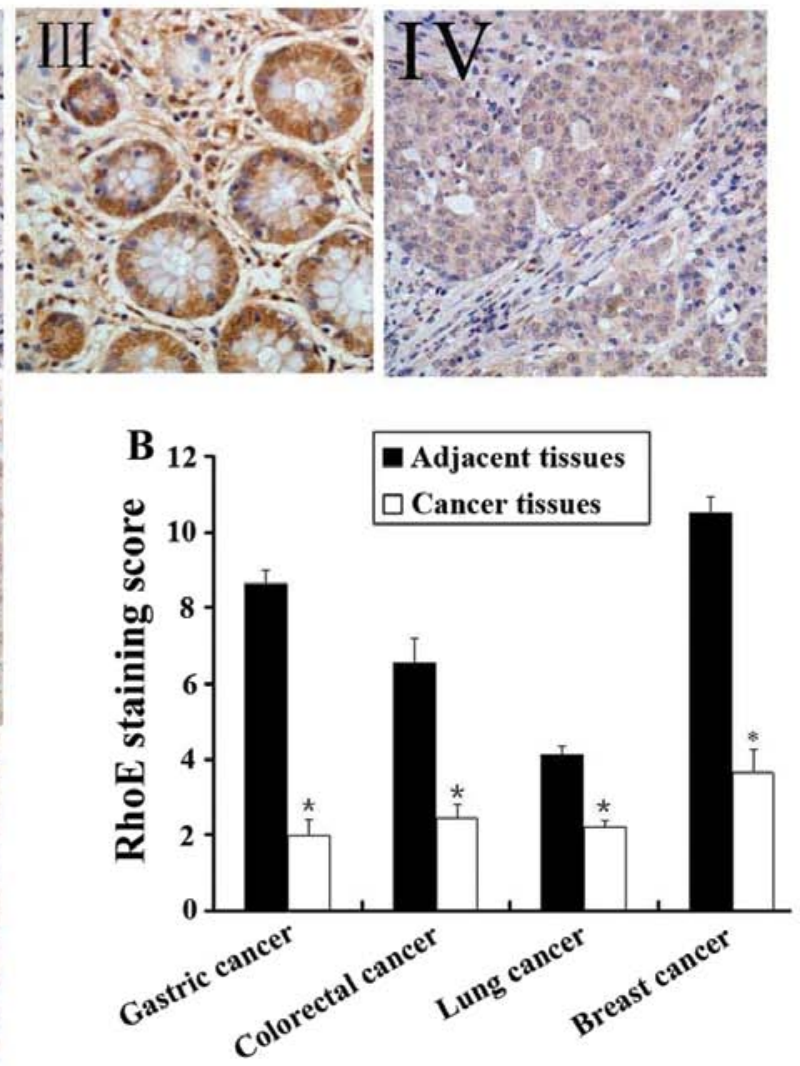

Figure 1. Downregulated RhoE expression in human cancer tissues. (A) AI, AIII, AV and AVII, respectively, represent the gastric, colorectal, lung and breast adjacent tissues showing strong immunosignals in the epithelial cells. AII, AIV, AVI and AVIII were the corresponding cancer tissues exhibiting obvious decline in the cancer cells $(20 \times 10)$. (B) The average staining score of RhoE in different cancers and adjacent tissues $\left({ }^{*} \mathrm{p}<0.05\right)$.

by western blot analysis in the ten different cancer cell lines. The data showed that RhoE protein expression was relatively higher in the cell lines HEK293, AGS, SMMC7721, LOVO, and A549, as well as NIH3T3 and COS7, while it was found at a relatively lower level in MKN45, SW480, HepG2 and SPCA1 cells (data not shown).

To explore the effects of RhoE on proliferation and invasion activities of cancer cells, the four RhoE low-expressed cell lines, MKN45, SW480, HepG2 and SPCA1, were transfected with pCDNA3.0-RhoE or pCDNA3.0, and the expression level of RhoE was detected by western blot analysis (Fig. 2B). We found that the RhoE expressing cells displayed significantly reduced capability to invade through Matrigel-coated Transwells (Fig. 2A). Further, the growth rate of RhoE transfected cancer cell lines, in each case, was significantly slower than that of the control cells and the parental cells (Fig. 3). These data suggest that RhoE expression can inhibit cell proliferation and suppresses invasion of the cancer cells.

Forced RhoE expression in cancer cells induces cell cycle arrest and apoptosis. To assess if $\mathrm{RhoE}$ expression affects cancer cell proliferation by modulating cell cycle progression and/or survival, the RhoE transfectants were analyzed by fluorescence-activated cell sorting (FACS). The results showed that the percentage of G1 phase cells increased significantly with RhoE overexpression. Contrary to the empty plasmid transfected control cells, the RhoE overexpressed cells failed to transit through the G1/S check point when they were stimulated by $10 \%$ serum (Fig. 4A). The TUNEL analysis and the Hoechest/PI stained cells also showed that the apoptosis rate of the RhoE expressing cells was significantly higher than that of the controls $(\mathrm{p}<0.01)$ (Fig. 4B). Similar observations were made by visual imaging of caspase- 3 positive cells (data not shown). These results provide evidence that RhoE overexpression not only induces cell growth arrest through inhibiting the G1/S phase transition, but also promotes apoptosis in the cancer cells.

RhoE expression is regulated by p53 transcription activity in cancer cell lines. It was reported that RhoE promoter contained several p53-binding sites and RhoE was one of the p53 transcriptional target genes (9). At the beginning of dissecting the mechanism of RhoE regulation in cancer, we determined a potential correlation of endogenous RhoE expression with p21-promoter-luc activities driven by cellular p53 in MKN45, SPCA1, SW480 and HepG2 cancer cell lines which also showed a relatively lower RhoE expression, and the luciferase/Renilla (Luc/Ren) ratios were significantly higher in cells with relatively high RhoE expression $(\mathrm{p}<0.05)$ (Fig. 5A). These results suggest that RhoE expression could be associated with endogenous p53 transcription activity in the absence of the genotoxic stress. 

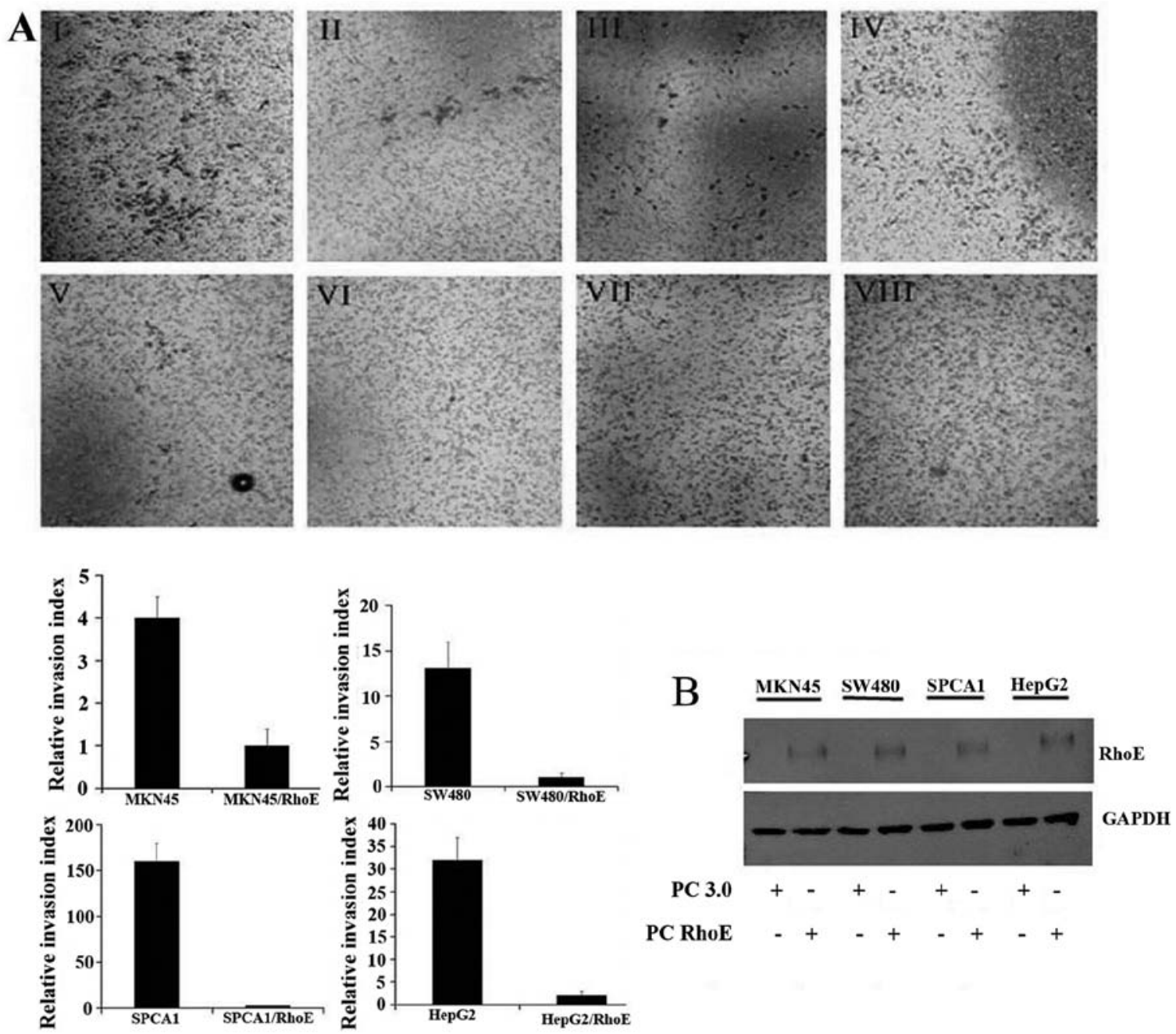

Figure 2. Enhanced RhoE expression inhibits cancer cells invasion. (A) The invasion activity of cells was assayed in Matrigel-coated transwells and cells that succeeded in invading the Matrigel were quantified $48 \mathrm{~h}$ after plating, and then images were taken. AI-IV were MKN45, SW480, SPCA1 and HepG2, respectively, transfectants with pcDNA3.0. AV-VIII were the corresponding pcDNA3.0-RhoE transfectants. Results are presented as mean $\pm \mathrm{SEM}\left(\mathrm{n}=3,{ }^{*} \mathrm{p}<0.05\right)$. Magnification, x200. (B) Immunoblot analysis of RhoE expression in four RhoE low-expressed cell lines (SPCA1, SW480, HepG2 and MKN45) that were, respectively, transfected with pcDNA3.0 and pcDNA3.0-RhoE. GAPDH was used as a loading control.

To determine whether forced $\mathrm{p} 53$ protein expression could cause the protein expression of RhoE, plasmids expressing PEGFP-wt-p53 or PEGFP-empty were transiently transfected into 4 cell lines with relatively low RhoE levels. Transfection efficiency was determined by counting the number of GFP-expressing cells per randomly chosen field of 100 cells at $24 \mathrm{~h}$ after transfection (multiple fields), and the mean number was determined as $45 \%$. RhoE expression level was detected by western blot analysis while the transfection efficiency was monitored by GFP expression (Fig. 5B). The results revealed that the endogenous RhoE expression was increased significantly upon p53 expression in these cells. These results provide evidence that RhoE might be subject to p53 transcription regulation.

p53 mutation decreases the activity of RhoE promoter. To determine whether low RhoE expression was the effect of p53 mutation or deletion, we sequenced the coding regions of the endogenous p53 gene by RT-PCR in these RhoE low-expressed cancer cell lines. However, we only found one meaningful mutation in SW480 cells (codon 273) in which both the activity of p53 and the expression level of RhoE were lowest among the four cancer cell lines. These results indicated the possibility that the decreased p53 activity is related to the detected mutations in the cancer cells.

To confirm if p53 mutation could affect the RhoE expression, we generated EGFP-mt-p53 constructs which contained p53 hot point mutations $(175,248,273,282,175 / 248,175 / 273$, $175 / 282,248 / 273,248 / 282,273 / 282)$. These mutants together with RhoE-promoter-3000-luc and PRL-TK were co-transfected into PC3 cells (p53 null) and P21-promoter-luc was taken as positive control. We found that Luc/Ren ratios were significantly decreased in the cells which were transfected with EGFP-mt-p53 compared with that of EGFP-wt-p53. However, there was no difference between the mutants (Fig. 5C). These results suggest that the p53 gene status is also a key factor affecting RhoE expression and p53 mutation could partly account for RhoE low expression. 

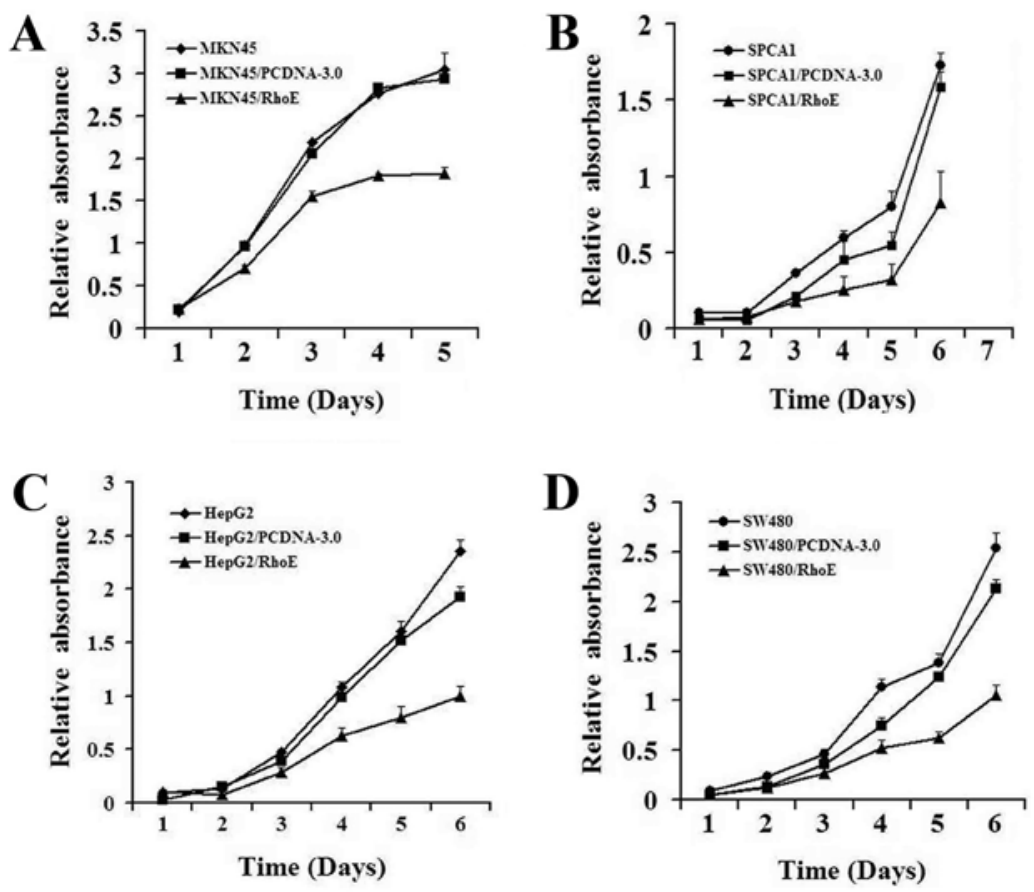

Figure 3. RhoE regulates cancer cells proliferation. (A) MKN45, (B) SPCA1, (C) HepG2 and (D) SW480 were stably transfected with pcDNA3.0 and pcDNA3.0RhoE, respectively. Monolayer growth rates of cells were determined by CCK 8 assay. Results are presented as mean \pm SEM $\left(n=3,{ }^{*} p<0.05\right)$.
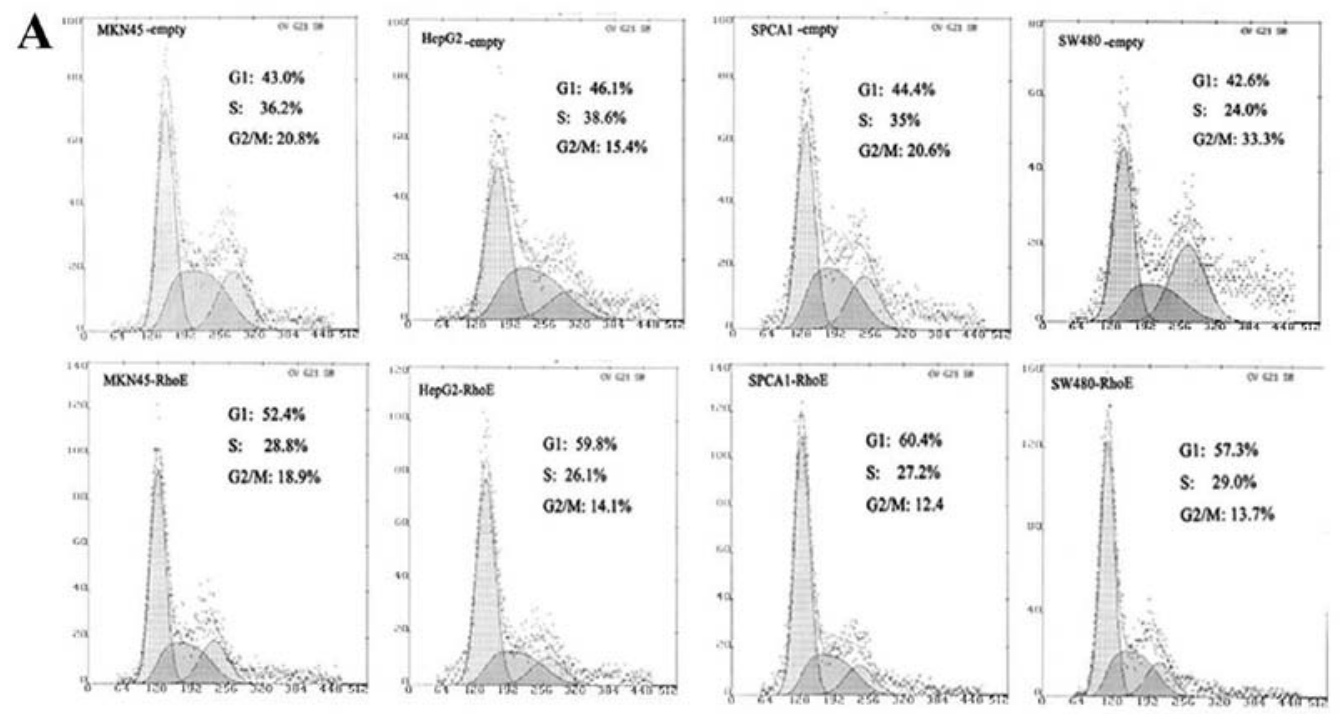

B
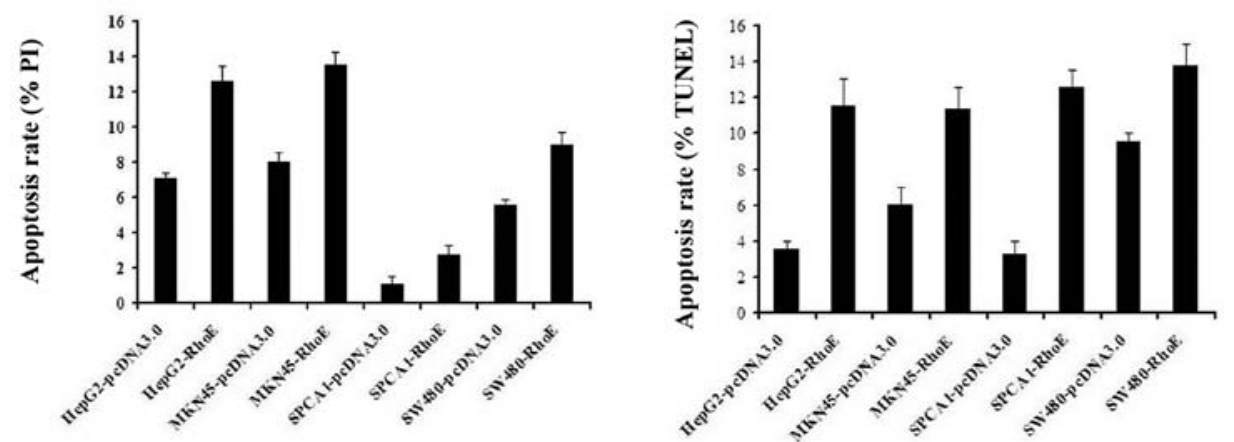

Figure 4. RhoE induces G1-phase arrest and apoptosis. (A) MKN45, HepG2, SPCA1 and SW480 cells which were transfected with pcDNA3.0 or pcDNA3.0-RhoE were starved for $24 \mathrm{~h}$ in $0.5 \%$ serum-containing medium and then were stimulated with $10 \%$ FBS for $24 \mathrm{~h}$. Their DNA contents were analyzed by flow cytometry as described in Materials and methods. Results are presented as mean \pm SEM ( $n=3, p<0.05)$. (B) Apoptosis rate was determined by Hoechst/PI staining and TUNEL experiments as described in Materials and methods. Results are presented as mean $\pm \operatorname{SEM}(n=3, p<0.05)$. 


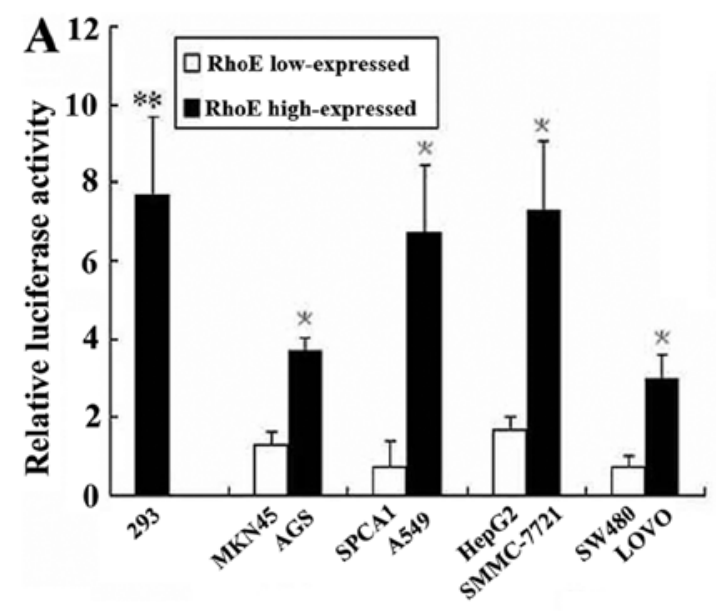

B
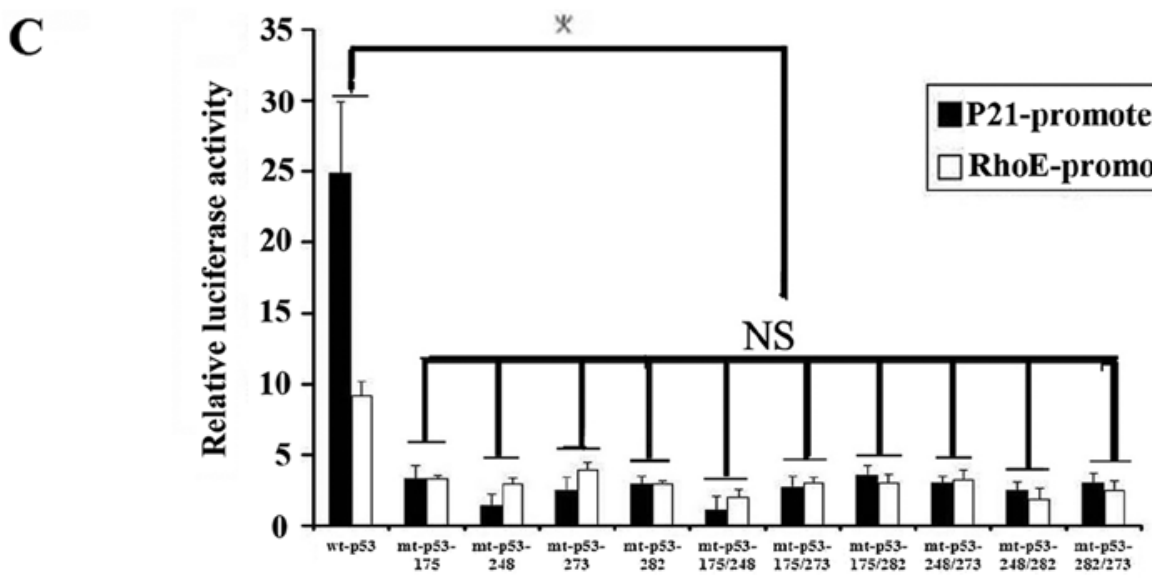

Figure 5. RhoE transcriptional activity is increased by wild p53 and decreased by p53 mutation. (A) P53 activity decreased in the RhoE low-expressing cell lines. All of the four cell lines were co-transfected transiently with the p53 reporter (p21-promoter-luc) and the internal parameter (PRL-TK) construct. The samples were collected after 48-h transfection. Error bars represent the standard errors of the means, and the asterisk ( $\left.{ }^{*}\right)$ indicates statistical difference of pairs of samples that are significant $(\mathrm{p}<0.05)$ while the NS indicates no statistical difference between normal cell lines and the cancer cell lines ( $>0.05)$. (B) PEGFP-wt-p53 and the PEGFP-empty were transiently transfected into the 4 cell lines which RhoE were low-expressed. Transfection efficiency was determined by counting the number of GFP-expressing cells per randomly chosen field of 100 cells at $24 \mathrm{~h}$ after transfection, and the mean number was determined as $45 \%$. Cells were collected and lysed after $48 \mathrm{~h}$ and RhoE expression was detected by western blot analysis. Overexpression of p53 significantly enhances RhoE protein level in the 4 cell lines. Results are presented as mean \pm SEM ( $n=3$, $\left.{ }^{*} \mathrm{p}<0.05\right)$ (C) P53 mutation decreased the activity of RhoE promoter. EGFP-mt-p53 constructs which contained p53 hot point mutations $(175,248,273,282,175 / 248,175 / 273,175 / 282,248 / 273,248 / 282,273 / 282)$ together with RhoE-promoter-3000-luc and PRL-TK were co-transfected into PC3 cells (p53 null). P21-promoter-luc was taken as positive control. Luc/Ren ratios were significantly decreased in the cells which were transfected with EGFP-mt-p53 than that of EGFP-wt-p53, but there was no difference among the mutants. Results are presented as mean \pm SEM $\left(n=3,{ }^{*} \mathrm{p}<0.05\right)$.

\section{Discussion}

Rho GTPases are important regulators of cell cytoskeleton organization and gene transcription, and deregulation of Rho family members has been shown to closely associate with the malignant phenotypes of cancer $(18,19)$. While most Rho proteins are geranylgeranylated and are subject to GTP-hydrolysis regulation, RhoE is farnesylated and does not act as a classical GTPase switch without a detectable GTPase activity (6). These biochemical findings suggest that RhoE may employ a unique regulatory mechanism and exhibits different function from other Rho family members.

RhoA has been implicated in the regulation of cell morphology, motility, transformation, proliferation and tumorigenicity (20-23). Previous studies suggest that RhoE can function as an antagonist of RhoA signaling in two ways: one is to bind directly to ROCK1 (a RhoA target effector) and inhibit its activity; the other is to interact with p190B
RhoGAP (a RhoA negative regulator) to reduce cellular level of RhoA-GTP $(18,24,25)$. Since RhoA is likely to play a positive role in cancer progression, we propose that RhoE might work in an opposite way. In our previous study, we found that RhoA was highly expressed in gastric cancer tissues and RhoA-specific small interfering RNA could partially reversed the malignant phenotypes of gastric cancer (26). There are several studies on RhoE, and substantial research shows RhoE is downregulated in most cancers $(7,11,27-29)$, and there are also some studies indicated that RhoE is overexpressed and acts as a metastasis promoter in certain cancers $(10,12)$. Here, we have evaluated RhoE protein expression patterns in four types of human cancer that are compared with the matched, adjacent tissues by immunohistochemistry, and the results showed that RhoE expression levels decreased significantly in each cancer, and in some cases RhoE appears to be completely absent from the tumors. Additionally, we found that RhoE expression level was inversely associated with the tumor differentiation grade. The 
differences seem to be more significant in breast and gastric cancer tissues, and less in lung cancer tissues.

To explore the effect of enhanced RhoE expression on the phenotypes of cancer cells, four cancer cell lines (MKN45, HepG2, SW480 and SPCA1) which show relatively lower RhoE expression were transfected with pcDNA3.0-RhoE. Overexpression of RhoE not only significantly inhibited the cancer cell proliferation, migration and invasion, but also induced apoptosis. These observations indicate that RhoE is intimately involved in many aspects of tumor progression and suggest the possibility that RhoE and its signaling cascade could serve as potential therapeutic target in anticancer treatment.

Since RhoE has a prominent effect on cancer cell proliferation, we thought that it might be involved in cell cycle and/or survival regulation. Our FACS analysis has shown that enhanced RhoE expression inhibited G1/S cell cycle transition and promoted apoptosis in the four tested cell lines. Previously, Villalonga et al (9) and Poch et al (30) also reported that RhoE could block G1 phase cell cycle progression in NIH3T3 cells and human glioblastoma U87 cells, while Bektic et al (31) found that RhoE could also induce a G2 block in prostate cancer cells $(31,32)$. The differences may be due to different cancer types or cell lines used in the experiments.

Our unpublished data show that RhoE level does not change in response to serum stimulation. Since the study of Ongusaha et al indicated that RhoE was a pro-survival target gene of p53 which inhibits ROCK1-mediated apoptosis in response to genotoxic stress (16), we subsequently investigated the p53 activity by dual-luciferase reporter assay, and found that the transcription activity of p53 was low in the RhoE lowexpressed cancer cell lines and high in the high RhoE expressed cancer cell lines. Furthermore, in each of the four examined cell lines with relatively low RhoE expressions (MKN45, HepG2, SW480 and SPCA1), we also found exogenous p53 expression significantly enhanced the RhoE expression. Taken together, these findings confirm that the expression of RhoE was regulated by $\mathrm{p} 53$.

p53 activity can be regulated by numerous factors in cells, and loss of function mutations is prominent in the reduction of its tumor surveillance function.p53 gene mutation was found in $>50 \%$ of all types of human cancers and $>80 \%$ were missense mutations that lead to the synthesis of a stable full-length protein (33). At the beginning of understanding the reason of the significant differences of p53 activities in different cancer cell lines, we have examined p53 mutations in 9 cancer cell lines and found only one meaningful mutation in SW480, and the activity of p53 and the expression level of RhoE were both the lowest in this cell line. These results, coupled with the demonstration that increased functional p53 expression could increase RhoE protein level in cells, suggest that p53 mutations in cancer cells may be related to the low expression of RhoE. To demonstrate this hypothesis, 10 kinds of PEGFP-mt-p53 vectors with the hot points of p53 mutation were generated. We found that Luc/Ren ratios were significantly lower in the cells which were transfected with EGFP-mt-p53 than that of EGFP-wt-p53, and there was no difference between the mutants. These results suggest that RhoE expression could not only be associated with p53 transcription but also be affected by 553 mutation.
Previous cell biology studies have suggested that RhoE was able to bind to ROCK1 and to inhibit ROCK-induced myosin phosphatase phosphorylation (25). ROCK1 is a RhoA downstream target known to mediate actin stress fiber assembly, whereas RhoE was found to antagonize RhoA to induce stress fiber disassembly $(18,19)$. RhoE could also be phosphorylated by ROCK1, leading to an increased stability and altered cellular localization. Another study has shown that expression of RhoE leads to decreased cellular RhoA-GTP by increasing the GAP activity of p190B RhoGAP (18). Here we have shown that RhoE expression can be regulated by $\mathrm{p} 53$ transcription activity. We have verified that $\mathrm{p}-\mathrm{MLC}$ level, an indicator of endogenous RhoA-ROCK signal, decreased in the RhoE-transfectant cancer cells (data not shown). Therefore, it is possible that RhoE mediate signals from p53 and serves as an antagonism of RhoA signaling in these cancer cells through a direct or indirect interaction with p190B RhoGAP or ROCK1.

In summary, RhoE expression was found significantly reduced in human gastric, colorectal, lung and breast carcinoma tissues compared to that of the adjacent normal tissues. Increased RhoE expression in these cancer cell lines resulted in an inhibition of proliferation, migration and invasion, and induced cell apoptosis. RhoE expression could be regulated by p53 transcription and decreased by p53 mutation. Our findings strongly suggest that RhoE can act as a candidate tumor suppressor and serve as a marker of cancer classification or progression.

\section{Acknowledgements}

This study was supported by the National Natural Science Foundation of China (nos. 81071640 and 30672378), the National Basic Research Program of China (2011CB935800) and the Funds of Health Department of Sichuan Province (no. 4241218R).

\section{References}

1. Aznar S, Valeron PF, Rincon SV, Perez LF, Perona R and Lacal JC: Simultaneous tyrosine and serine phosphorylation of Stat 3 transcription factor is involved in a Rho GTPase oncogenic transformation. Mol Biol Cell 12: 3282-3294, 2001.

2. Mackay D J and Hall A: Rho GTPases. J Biol Chem 273: 20685-20688, 1998.

3. Sahai E and Marshall CJ: Rho-GTPases and cancer. Nat Rev Cancer 2: 133-142, 2002.

4. Van Aelst L and D'Souza-Schorey C: Rho GTPases and signaling networks. Gene Dev 11: 2295-2322, 1997.

5. Pruitt $\mathrm{K}$ and Der $\mathrm{CJ}$ : Ras and rho regulation of the cell cycle and oncogenesis. Cancer Lett 171: 1-10, 2001.

6. Foster R, Hu KQ, Lu Y, Nolan KM, Thissen J and Settleman J: Identification of a novel human rho protein with unusual properties: GTPase deficiency and in vivo farnesylation. Mol Cell Biol 16: 2689-2699, 1996.

7. Nobes CD, Lauritzen I, Mattei MG, Paris S, Hall A and Chardin P: A new member of the Rho family, Rnd1, promotes disassembly of actin filament structures and loss of cell adhesion. J Cell Biol 141: 187-197, 1998.

8. Riento K, Totty N, Villalonga P, Garg R, Guasch R and Ridley AJ: RhoE function is regulated by Rock 1-mediated phosphorylation. EMBO J 24: 1170-1180, 2005.

9. Villalonga P, Guasch RM, Riento K and Ridley AJ: RhoE inhibits cell cycle progression and ras-induced transformation. Mol Cell Biol 24: 7829-7840, 2004.

10. Cuiyan Z, Jie H, Fang Z, Kezhi Z, Junting W, Susheng S, Xiaoli F, Ning L, Xinhua $M$ and Zhaoli C: Overexpression of RhoE in non-small cell lung cancer (NSCLC) is associated with smoking and correlates with dna copy number changes. Cancer Biol Ther 6: 335-342, 2007. 
11. Ma W, Wong CC, Tung EK, Wong CM and Ng IO: RhoE is frequently down-regulated in hepatocellular carcinoma (HCC) and suppresses HCC invasion through antagonizing the Rho/Rho-Kinase/Myosin phosphatase target pathway. Hepatology 57: 152-161, 2013.

12. Katiyar P and Aplin AE: FOXD3 regulates migration properties and Rnd3 expression in melanoma cells. Mol Cancer Res 9: 545-552, 2011.

13. Lowe SW, Schmitt EM, Smith SW, Osborne BA and Jacks T: p53 is required for radiation-induced apoptosis in mouse thymocytes. Nature 362: 847-849, 1993.

14. Lane DP: Cancer. p53, guardian of the genome. Nature 358 : $15-16,1992$.

15. Clarke AR, Purdie CA, Harrison DJ, Morris RG, Bird CC Hooper ML and Wyllie AH: Thymocyte apoptosis induced by p53-dependent and independent pathways. Nature 362: 849-852, 1993

16. Ongusaha PP, Kim HG, Boswell SA, Ridley AJ, Der CJ, Dotto GP, Kim YB, Aaronson SA and Lee SW: RhoE is a pro-survival p53 target gene that inhibits ROCK1-mediated apoptosis in response to genotoxic stress. Curr Biol 16 2466-2472, 2006.

17. Liu Y and Bodmer WF: Analysis of p53 mutations and their expression in 56 colorectal cancer cell lines. Proc Natl Acad Sci USA 103: 976-981, 2006.

18.Wennerberg K, Forget MA, Ellerbroek SM, Arthur WT, Burridge K, Settleman J, Der CJ and Hansen SH: Rnd proteins function as RhoA antagonists by activating p190 RhoGAP. Curr Biol 13: 1106-1115, 2003.

19. Etienne-Manneville $S$ and Hall A: Rho GTPases in cell biology. Nature 420: 629-635, 2002.

20. Li X, Liu L, Tupper JC, Bannerman DD, Winn RK, Sebti SM, Hamilton AD and Harlan JM: Inhibition of protein geranylgeranylation and RhoA/RhoA kinase pathway induces apoptosis in human endothelial cells. J Biol Chem 277: 15309-15316, 2002.

21. Ghosh PM, Ghosh-Choudhury N, Moyer ML, Mott GE, Thomas CA, Foster BA, Greenberg NM and Kreisberg JI: Role of RhoA activation in the growth and morphology of a murine prostate tumor cell line. Oncogene 18: 4120-4130, 1999.

22. Kamai T, Kawakami S, Koga F, Arai G, Takagi K, Arai K, Tsujii T and Yoshida KI: RhoA is associated with invasion and lymph node metastasis in upper urinary tract cancer. BJU Int 91: 234-238, 2003.
23. Yoshioka K, Nakamori S and Itoh K: Overexpression of small GTP-binding protein RhoA promotes invasion of tumor cells. Cancer Res 59: 2004-2010, 1999.

24. Guasch RM, Scambler P, Jones GE and Ridley AJ: RhoE regulates actin cytoskeleton organization and cell migration. Mol Cell Biol 18: 4761-4771, 1998.

25. Riento K, Guasch RM, Garg R, Jin B and Ridley AJ: RhoE binds to ROCK1 and inhibits downstream signaling. Mol Cell Biol 23: 4219-4229, 2003

26. Liu N, Bi F, Pan Y, Sun L, Xue Y, Shi Y, Yao X, Zheng Y and Fan D: Reversal of the malignant phenotype of gastric cancer cells by inhibition of RhoA expression and activity. Clin Cancer Res 10: 6239-6247, 2004

27. Grise F, Sena S and Bidaud-Meynard A: Rnd3/RhoE is down-regulated in hepatocellular carcinoma and controls cellular invasion. Hepatology 55: 1766-1775, 2012.

28. Luo H, Dong Z, Zou J, Zeng Q, Wu D and Liu L: Downregulation of $\mathrm{RhoE}$ is associated with progression and poor prognosis in hepatocellular carcinoma. J Surg Oncol 105: 699-704, 2012

29. Zhao H, Yang J, Fan T, Li S and Ren X: RhoE functions as a tumor suppressor in esophageal squamous cell carcinoma and modulates the PTEN/PI3K/Akt signaling pathway. Tumour Biol 33: 1363-1374, 2012.

30. Poch E, Mi ambres R, Mocholí E, Ivorra C, Pérez-Aragó A Guerri C, Pérez-Roger I and Guasch RM: RhoE interferes with $\mathrm{Rb}$ inactivation and regulates the proliferation and survival of the U87 human glioblastoma cell line. Exp Cell Res 313: 719-731, 2007

31. Bektic J, Pfeil K, Berger AP, Ramoner R, Pelzer A, Schafer G, Kofler K, Bartsch G and Klocker H: Small G-protein RhoE is underexpressed in prostate cancer and induces cell cycle arrest and apoptosis. Prostate 64: 332-340, 2005.

32. Trojan L, Schaaf A, Steidler A, Haak M, Thalmann G, Knoll T, Gretz N, Alken P and Michel MS: Identification of metastasis-associated genes in prostate cancer by genetic profiling of human prostate cancer cell lines. Anticancer Res 25: 183-191, 2005.

33. Soussi T and Béroud C: Assessing TP53 status in human tumours to evaluate clinical outcome. Nat Rev Cancer 1: 233-239, 2001. 